\title{
Low Back Pain After Nonspinal Surgery: The Characteristics of Presumed Lumbar Paraspinal Compartment Syndrome
}

\author{
Andrew J. Haig, MD, Anne G. Hartigan, MD, Douglas Quint, MD \\ INTRODUCTION
}

For persons who undergo general surgical procedures, there are a number of potential causes of early postoperative back pain. Intra-abdominal causes generally include peritonitis and kidney infection but may also include disorders related to the specific operation such as aneurysm, stone formation, etc. Intraoperative positioning, inactivity, and premorbid back conditions often make postoperative back pain a complex issue.

Compartment syndromes can occur after surgery, but they are almost always found in the limbs, not the spine $[1,2]$. Although the paraspinal muscles are compartmentalized by fascia, a compartment syndrome of the paraspinal muscles has only been reported in a handful of cases [3-6]. The authors report on 2 cases of postoperative paraspinal muscle scarring and sensory nerve damage that may represent a compartment syndrome. This first report of a possibly pathognomonic physical examination finding (ie, paraspinal sensory loss), electromyogram findings, and progression of abnormal magnetic resonance imaging (MRI) findings may help spine practitioners recognize and treat this rare complication of general surgery. This report was exempted from review by the university's Institutional Review Board.

\section{PARASPINAL ELECTROMYOGRAPHY METHODS}

Electrodiagnostic testing was performed by A.J.H., an American Board of Electrodiagnostic Medicine board-certified specialist, on a Viking IV EMG machine (Nicolet Biomedical, Madison, WI) using a monopolar needle. Codified and quantified techniques were used for EMG of the lumbar paraspinal muscles. The following terminology used in this report is clarified. The term "paraspinal mapping" was originally used for a very extensive protocol which inserted a 50-mm needle to its hub in 45 different directions and accurately localized the multifidus, longissimus, and iliocostalis muscles [7]. MiniPM is an abbreviated protocol that only explored the multifidus muscle with 12 needle insertions [8]. However, MiniPM is now commonly referred to as paraspinal mapping. Because both protocols were used in this study, paraspinal mapping is the term used for the extensive protocol and MiniPM for the abbreviated protocol.

The reader is referred to extensive documentation of the paraspinal mapping and MiniPM protocols $[7,8]$. In brief, MiniPM involves marking 4 locations that are $2.5 \mathrm{~cm}$ lateral to the spinous processes of L3, L4, L5, and S1. A 50- to 75-mm monopolar needle is inserted at a $45^{\circ}$ angle to the surface and directly toward the spinous process, then withdrawn, redirected cranially and medially $45^{\circ}$ to the bone, then withdrawn and redirected caudally and medially $45^{\circ}$ to the bone, with 5 -mm insertions. Spontaneous activity is scored 0-4+ for both the medial and lateral part of the insertion. This results in 12 needle insertions and 24 scores, with a theoretical maximum score of 96 . The range of normal for asymptomatic younger persons is $0-2$.

Paraspinal mapping adds insertions at a line parallel to the spine, halfway between the spine and the posterior superior iliac spine, to sample the longissimus. Additional insertions half way between the lateral border of the erector spinae and the midline accurately sample the iliocostalis. Finally, an area of insertion similar to MiniPM, but at an additional level 2.5 $\mathrm{cm}$ caudal to the sacral base is included. For the original paraspinal mapping protocol norms
A.J.H. Department of Physical Medicine and Rehabilitation, The University of Michigan, 325 E Eisenhower, Ann Arbor, Ml 48108. Address correspondence to: A.J.H.; e-mail: andyhaig@umich.edu

Disclosure: nothing to disclose

A.G.H. Department of Physical Medicine and Rehabilitation, The University of Michigan, Ann Arbor, MI.

Disclosure: nothing to disclose

D.Q. Department of Radiology, The University of Michigan, Ann Arbor, MI. Disclosure: nothing to disclose

Disclosure Key can be found on the Table of Contents and at www.pmrjournal.org

Submitted for publication February 23, 2008; accepted December 13, 2008. 


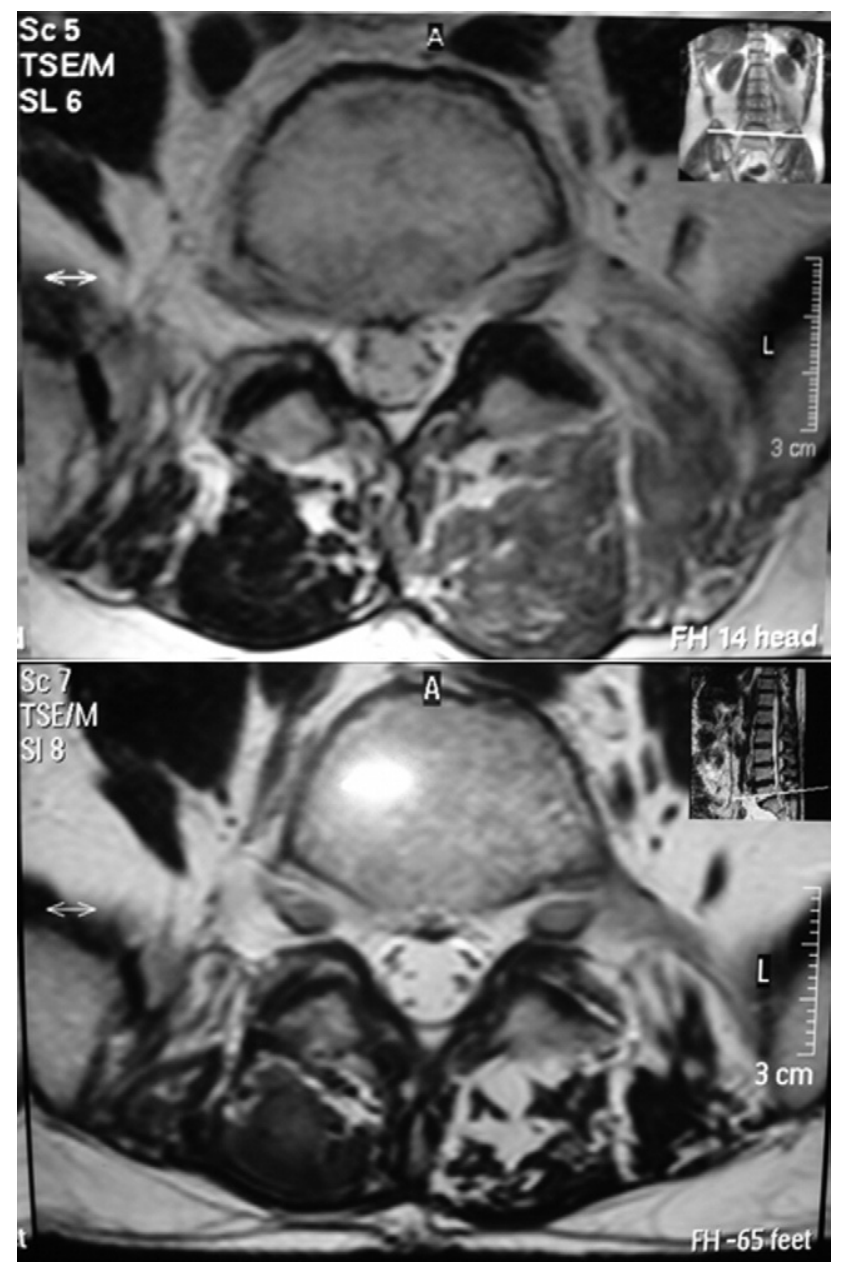

Figure 1. Case 1: Axial T2-weighted lumbosacral spine MRI obtained 2 days after surgery (top) and 10 months later (bottom) showing suspected paraspinal compartment syndrome. Initially (top image), increased T2 signal from the left paraspinal muscles compared to the right consistent with edema is observed. Follow-up axial T2-weighted MR imaging (bottom) shows left paraspinal muscle atrophy with fatty replacement.

are 0-5. The techniques of MiniPM and paraspinal mapping are standardized for L2 through L5 paraspinals, but in this study they are also used in the thoracic paraspinals.

\section{CASE REPORTS}

\section{Case 1}

A 57-year-old man awoke from an otherwise uneventful aortoiliac bypass procedure with excruciating low back pain. Magnetic resonance imaging (MRI) performed 2 days later was interpreted as demonstrating a disk bulge or small herniation. The consulting orthopedic surgeon recommended that the patient have a diskectomy once he recovered from the initial surgery. His symptoms persisted with severe pain and disability. Ten months later he presented to the spine clinic for a second opinion regarding diskectomy.
He described low back pain with sitting, standing, and lifting. There was no worsening of pain at night, no sciatica, and no bowel or bladder involvement. The initial spine clinic examination was remarkable for superficial sensory loss to pinprick and light touch in his low back, in a reproducible area from $9 \mathrm{~cm}$ caudal to $17 \mathrm{~cm}$ cranial to the lumbosacral junction, and symmetrically $23 \mathrm{~cm}$ wide. There was diffuse deep tenderness in the lumbar region. The left patellar and medial hamstring reflexes and both Achilles reflexes were absent, but no strength deficit was appreciated.

In retrospect, there was a marked difference between the left and right paraspinal muscle signal on the initial MRI (Figure 1; top) with increased T2 signal found in the left paraspinal muscle (multifidus, longissimus, and iliocostalis muscles) region. A repeated lumbosacral MRI study performed 11 months after surgery (Figure 1; bottom) showed atrophic left paraspinal musculature with fatty replacement. Sagittal para-midline slices showed what the authors have come to recognize as a characteristic cranial-caudal distribution of signal change, possibly surrounded by a higherdensity capsule, with either spared peripheral paraspinal musculature or residual fascial lining (Figure 2). The iliopsoas was notably normal in appearance.

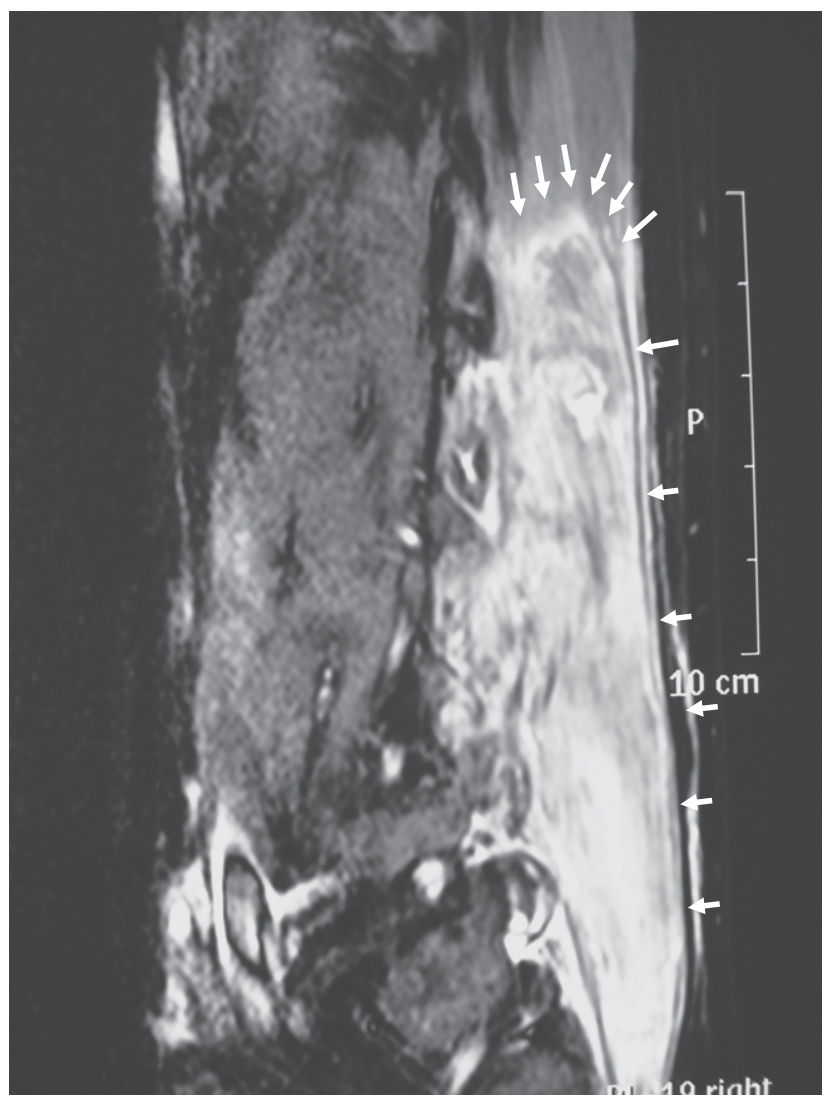

Figure 2. Case 1, parasagittal $\mathrm{T} 1$-weighted lumbosacral spine MRI at 10-month follow-up. The white arrows outline the area of altered tissue extending cranially to about T12, similar in shape and extent to Patient 2, as noted in Figure 3, and consistent with the extent of paraspinal EMG findings. 
Table 1. Paraspinal mapping findings in patient 1

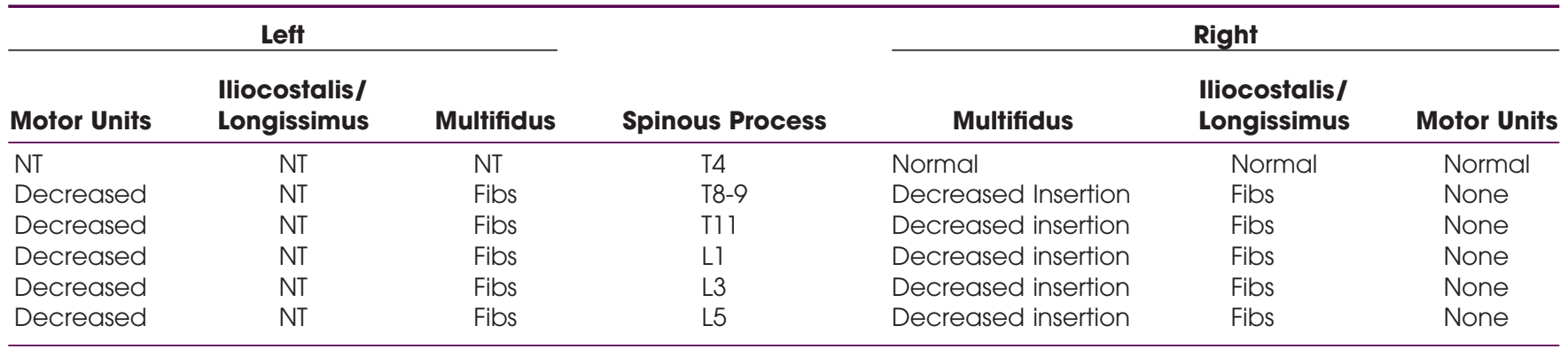

The "motor units" column represents motor unit findings in longissimus or multifidus, with "decreased" implying decreased recruitment. The "longissimus/ iliocostalis" and "multifidus" columns represent paraspinal mapping sites, with "decreased" implying decreased insertional activity (eg, fatty replacement) and "fibs" implying positive waves and fibrillations. There were no important differences between longissimus and iliocostalis. NT indicates not tested.

Because this is the first description of EMG in apparent paraspinal compartment syndrome, the technical findings are presented detail. Table 1 summarizes the needle EMG findings in the paraspinal muscles. In general there was severe muscle membrane instability caudally in the paraspinals with a fibrous feel to needle insertions and no recruitable motor units. This finding diminished cranially and laterally. The radiologically more affected left side was also more abnormal on EMG. Needle electromyography of 6 muscles (the tensor fascia lata, gluteus maximus, vastus medialis, peroneus longus, tibialis anterior, and medial gastrocnemius) in the left lower limb (the limb with the reflex changes on physical examination) was normal.

\section{Case 2}

A 34-year-old man awoke with low back pain after gastric bypass surgery. He did not have spinal anesthesia. He reported that serosanguineous drainage appeared from his coccygeal area days after surgery and continued for a month. Lumbosacral spine MRI performed at 1 month after surgery was interpreted as negative. One year later, the patient presented to the spine clinic with complete sensory loss to light touch and pinprick from the upper sacrum to the T11 level that extended bilaterally $15 \mathrm{~cm}$ from the midline. There were no lower-limb sensory, motor, or reflex abnormalities. Repeat lumbosacral MRI performed 1 year after surgery (Figure 3) demonstrated well-defined areas of abnormal signal within the paraspinal muscles bilaterally thought to represent possible areas of scarring. Peripheral paraspinal muscle sparing versus denser scar could not be ruled out on that study.

The EMG findings were largely symmetrical, but otherwise similar to the first case, with severe distal and medial damage normalizing by the level of T9. Specifically, multifidus muscles were explored bilaterally from the sacrum to the T9 level with the use of cranial extensions of the MiniPM technique. The earlier version of paraspinal mapping was used to explore the longissimus and iliocostalis muscles at the L3 level. In the lower lumbar region there was a very tough fibrous feel with a few positive waves. Despite good voluntary effort as evidenced by activity of complimentary
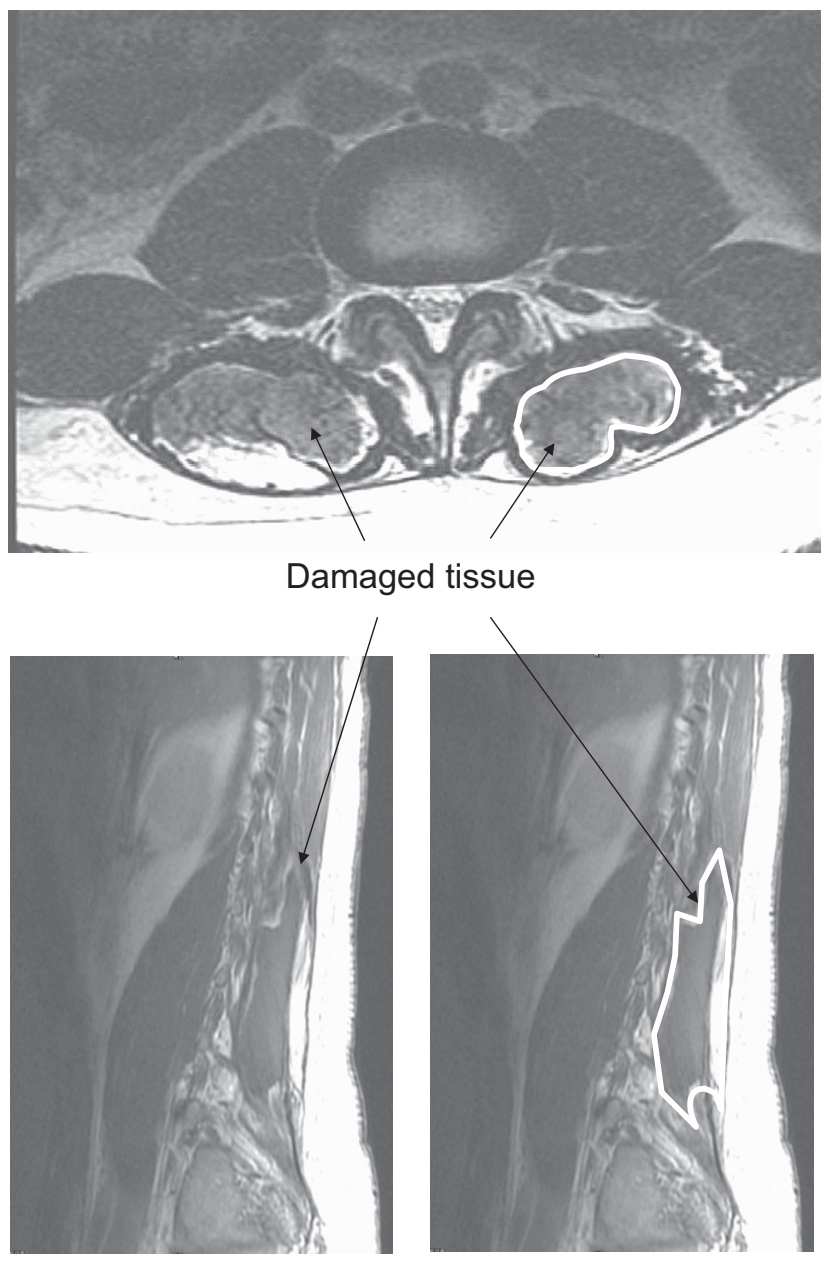

Figure 3. Case 2: Axial (top) and sagittal (bottom; annotated on the right) T2-weighted scans 1 year after surgery. Presumed scar has replaced the majority of the paraspinal muscles bilaterally (outlined by white line). No muscle atrophy or diffuse fatty replacement is seen. Spared paraspinal musculature vs. frank scar is again seen surrounding affected paraspinal muscles-thicker anteriorly than posteriorly. The apparently typical cephalocaudad distribution of these scarred muscles is wellseen on the sagittal images (bottom). 


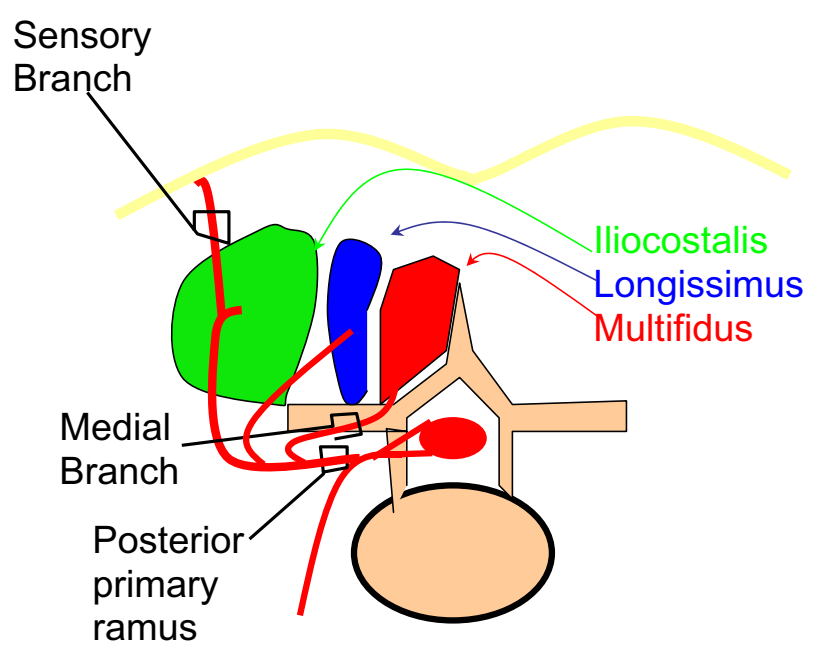

Figure 4. Schematic of the paraspinal compartment delineating innervation of the paraspinal muscles and overlying skin. Note that the hallmark finding of paraspinal skin sensory loss will not occur with isolated medial branch lesions from surgery or radiofrequency medial branch ablation.

muscles, no motor units were recruited in the multifidus, longissimus, or iliocostalis. In the upper lumbar region positive waves and fibrillations (2-3+) with small amplitude, long polyphasics (400 $\mu \mathrm{v}, 12-15 \mathrm{~ms}$ ) were seen. Needle examination of the thoracic paraspinals was normal above the T9 level. Six left leg muscles (the same as examined in patient 1), sural sensory conduction, and peroneal motor conduction studies were normal.

\section{DISCUSSION}

These 2 cases likely represent examples of the paraspinal compartment syndrome. To the authors' knowledge, this report is the first to demonstrate acute MRI findings, MRI changes over time, EMG findings, and the clinical finding of paraspinal sensory loss that might be specific for this syndrome.

Paraspinal compartment syndrome has only rarely been reported. DiFazio et al [3] reported the first case of paraspinal compartment syndrome, which occurred after an event of severe exertion. Subsequently, a few postoperative cases also have been reported [4-6]. The failure of early diagnosis in these 2 cases suggests that this syndrome may be overlooked and could be more common than this literature suggests.

The finding of superficial paraspinal sensory loss, which may be critical to early detection of this syndrome, is reported. This degree of sensory loss requires damage to the lateral branch of the posterior primary ramus at multiple levels [9]. Figure 4 schematically reviews the neural innervation of the pertinent lumbosacral paraspinal muscles. In the authors' extensive experience in performing paraspinal EMG, focal paraspinal sensory loss has never been encountered aside from these cases. Therefore, the finding of paraspinal sensory loss might be pathognomonic for paraspinal compartment syndrome.

There is not another readily explainable mechanism for these findings. Theoretically, ischemia of a paraspinal region without focal extrinsic pressure could occur; however, such a lesion has never been reported. Some recovery of a posterior primary ramus neurologic distribution lesion after simple ischemia would be expected and the MRI-suggested peripheral ring of either spared paraspinal musculature or frank scar tissue would not be expected. Direct external pressure seems unlikely because there were no pressure sores on the skin over the spinous processes and because the paraspinal region has an extensive collateral arterial blood supply.

In contrast, lesions that are known to affect the medial branch, including an apparent entrapment syndrome known as the lumbar dorsal ramus syndrome and iatrogenic radiofrequency selective dorsal neurotomy do not affect sensation [10-12]. A disk herniation could cause paraspinal sensory loss; however, substantial overlap in sensory distribution from other root innervation would likely disguise paraspinal sensory changes after single-level nerve root damage. Spinal stenosis is a potential cause of multilevel paraspinal sensory loss, but the authors believe that sensory loss from stenosis would not be isolated to the medial paraspinal region only, and would only occur in the context of sensory loss in the leg and profound motor loss.

The MRI findings in the paraspinal musculature are striking. In patient 1 , the increased T2 signal of some of the paraspinal muscles (Figure 1; top) was clearly evident within days of onset of the patient's symptoms. This finding was not recognized on the initial reading. Because a small herniated disk was suggested to be present on this study, an orthopedic surgeon recommended a diskectomy. The case serves as a reminder that the specificity of MRI findings in the lumbosacral region with respect to patient symptoms remains poor and can be frankly misleading [13,14]. Follow-up MRI performed almost a year later (Figure 1; bottom) demonstrated atrophy with fatty replacement of left-sided paraspinal muscles with suggestion of either a ring of apparent scar tissue or peripherally spared paraspinal musculature. These MRI findings are different from those seen with the bent spine syndrome or paraspinal myopathy (fatty changes without ring of scar), with disuse atrophy (shrunken muscle mass with fatty interdigitations that can occur as a result of pain), or with denervation atrophy (radiologically similar to disuse atrophy) [15-18]. Infarction of the muscle without compartment syndrome is a possibility; however, we would have expected atrophy and either sensory sparing or sensory recovery after an infarction.

Paraspinal EMG findings are consistent with the diagnosis but could be missed by a less-than-astute electromyographer. Clinicians who do not use a quantified approach such as paraspinal mapping may find an area of abnormal spontaneous activity and assume it is the result of a radiculopathy without exploring elsewhere. When diffuse abnormal spontaneous activity is found in the lumbar region, the authors' protocol suggests that the paraspinal EMG exam be extended 
proximally. Decreased insertional activity is a more subtle finding than the increased insertional activity that occurs with denervation.

In contrast to the bent spine syndrome and simple deconditioning, a tough outer layer surrounding the muscle is appreciated in the chronic phase of paraspinal compartment syndrome. The distribution is primarily in the multifidus muscle, with some involvement of the more lateral longissimus muscle. This same distribution can also be found in atrophy or the bent spine syndrome. However, the iliocostalis muscle is relatively spared. This sparing is probably because that muscle is not innervated from lower lumbar roots. Also, this muscle is located lateral to the main fascia of the compartment, which surrounds the longissimus and multifidus muscles. Although motor unit abnormalities in the paraspinal muscles are striking, motor activity is not typically assessed in the complex paraspinal muscle groups.

The authors can only speculate on the etiology of the apparent compartment syndrome in these patients. Gourgiotis et al [2] reported postischemic reperfusion as one etiology of limb compartment syndromes. This is a plausible explanation. The serosanguinous drainage in patient 2 could have been old blood or the sequelae of rhabdomyolysis. No creatine phosphokinase levels were available. The asymmetrical distribution of paraspinal muscle involvement in patient 1 suggests a different focal mechanism. Perhaps a radicular artery was stretched and torn, or some aspect of positioning uniquely caused pressure that blocked venous return. The authors know of another case (not reported here) that led to lumbar paraspinal compartment syndrome caused by infiltration of fluid after a misplaced catheter apparently increased local compartmental pressure. This mechanism of injury has not been reported previously. Chemical damage from infiltrated medications could have been a cause or contributor to the compartment syndrome, but it is not known what medications were infused in that patient.

These patients were not evaluated in their acute or subacute phase. However, given the severity of disability and pain in their undiagnosed and subsequently untreated conditions, some comment should be made about early diagnosis and treatment. All 3 patients reported that they had acute severe pain temporally related to their respective procedures. Acute pain after surgery is not uncommon, with causes including kidney infection, aneurysm rupture, peritonitis, and passing of stones. It is likely that these patients presented like Ferreira's case [6], with severe tenderness, swelling, and rigidity of the paraspinal muscle mass (which might be interpreted as "spasm"), along with an elevated creatine kinase level. Ferreira did not note the paraspinal sensory loss that appears to be quite specific for this process.

Acutely, the paraspinal parasthesias would raise suspicion for the diagnosis and compartment pressure measurements would likely be diagnostic. The MRI may also be diagnostic. EMG theoretically would show electrical silence early and diffuse membrane instability later. Although paraspinal mapping was used here, the profound nature of the instability suggests that any routine paraspinal examination would likely identify this lesion. However, without a more systematic approach such as paraspinal mapping, the electromyographer might consider that the paraspinal denervation was related to a single level radiculopathy. In this study, the use of the old paraspinal mapping technique allowed demonstration of the involvement of the longissimus and iliocostalis muscle, but this more extensive technique is not necessarily useful in clinical diagnosis.

Given the experience with acute compartment syndromes elsewhere in the body, delaying diagnosis for even a day or 2 could result in irreversible muscle damage and ultimately disability, and perhaps even mortality from myoglobinemia $[1,2]$. When compartment pressure in the limbs approaches within $30 \mathrm{~mm} \mathrm{Hg}$ of arterial pressure, experts recommend fasciotomy within 12 hours of onset. In the future, a surgeon or physiatrist aware of this process might recognize the possibility of the presence of this syndrome because of the sensory complaint. Abnormal creatine phosphokinase levels and elevated compartment measures might lead to urgent paraspinal fasciotomy.

This apparent paraspinal compartment syndrome was severely and chronically disabling in these patients. Back muscle flexibility and strength are important life functions that were compromised. Preservation of paraspinal muscle function is known to be critical in avoiding long term disability after spinal surgery and presumably after this disorder $[19,20]$. Resultant contractures and positional compensation for weakness might result in hyperextension of the lumbar spine and resultant facet joint or spinous process mediated pain. In cases of back muscle inadequacy, biomechanical changes such as elimination of hip flexion contractures may be appropriate components of rehabilitation [21].

\section{CONCLUSION}

Severe back pain after general/abdominal surgical procedures may suggest paraspinal compartment syndrome. Paraspinal sensory loss may be specific for this syndrome. MRI and EMG can suggest and possibly confirm the disorder in the appropriate clinical setting. However, there should be a sense of urgency in considering pressure monitoring and decompression of the involved compartment, as an untreated lesion can progress to severe, chronic disability.

\section{REFERENCES}

1. Konstantakos EK, Dalstrom DJ, Nelles ME, Laughlin RT, Prayson MJ. Diagnosis and management of extremity compartment syndromes: An orthopaedic perspective. Am Surg 2007;73:1199-1209.

2. Gourgiotis S, Villias C, Germanos S, Foukas A, Ridolfini MP. Acute limb compartment syndrome: A review. J Surg Ed 2007;64:178-186.

3. DiFazio FA, Barth RA, Frymoyer JW. Acute lumbar paraspinal compartment syndrome. A case report. J Bone Jt Surg Am 1991;73:1101-1103.

4. Osamura N, Takahashi K, Endo M, Kurumaya H, Shima I. Lumbar paraspinal myonecrosis after abdominal vascular surgery. A case report. Spine 2000;25:1852-1854.

5. Sava J, Moelleken A, Waxman K. Cardiac arrest caused by reperfusion injury after lumbar paraspinal compartment syndrome. J Trauma Injury Infect Crit Care 1999;46:196-197. 
6. Ferreira J, Galle C, Aminian A, et al. Lumbar paraspinal rhabdomyolysis and compartment syndrome after abdominal aortic aneurysm repair. J Vasc Surg 2003;37:198-201.

7. Haig AJ, Talley C, Grobler LJ, LeBreck DB: Paraspinal mapping: Quantified needle electromyography in lumbar radiculopathy. Muscle Nerve 1993;16:477-484.

8. Haig AJ. Clinical experience with Paraspinal Mapping. II: A simplified technique that eliminates three-fourths of needle insertions. Arch Phys Med Rehabil 1997;78:1185-1190.

9. Bogduk N. A reappraisal of the anatomy of the human lumbar paraspinal. J Anat 1980;131:525-540.

10. Bogduk N. Lumbar dorsal ramus syndrome. Med J Aust 1980;2:537541.

11. Fisher MA, Kacr D, Houchin J. Electrodiagnostic examination, back pain, and entrapment of posterior rami. Electromyography Clin Neurophysiol 1985;25:187.

12. Dreyfuss $P$, Halbrook B, Pauza K, Joshi A, McLarty J, Bogduk $N$. Efficacy and validity of radiofrequency neurotomy for chronic lumbar zygapophysial joint pain. Spine 2000;25:1270-1277.

13. Boden SD, Davis DO, Dina TS, Patronas NJ, Wiesel SW. Abnormal magnetic-resonance scans of the lumbar spine in asymptomatic subjects. A prospective investigation. J Bone Jt Surg Am 1990;72: 403-408.
14. Haig AJ, Geisser ME, Tong HC, et al. Electromyographic and magnetic resonance imaging to predict lumbar stenosis, low-back pain, and no back symptoms. J Bone Jt Surg Am 2007;89:358-366.

15. Serratrice G, Pouget J, Pellissier JF. Bent spine syndrome. J Neurol Neurosurg Psychiatry 1996;60:51-54.

16. Danneels LA, Vanderstraeten GG, Cambier DC, et al. Effects of three different training modalities on the cross sectional area of the lumbar multifidus muscle in patients with chronic low back pain. Br J Sports Med 2001;35:186-191.

17. Fetzer A, Yarjanian J, Haig AJ, Yamakawa KS. Paraspinal atrophy vs. denervation in back pain, spinal stenosis, and controls. Poster 54. American Association of Neuromuscular and Electrodiagnostic Medicine 52nd Annual Scientific Meeting, Monterey, CA, September 21-24, 2005.

18. Campbell WW, Vasconcelos $O$, Laine FJ. Focal atrophy of the multifidus muscle in lumbosacral radiculopathy. Muscle Nerve 1998;21: 1350-1353.

19. Keyserling M, Sudarsen P, Martin BJ, Haig AJ, Armstrong TJ. Effects of low back disability status on lower back discomfort during sustained and cyclical trunk flexion. Ergonomics 2005;48:219-233.

20. Sihvonen T, Herno A, Paljarvi L, Airaksinen O, Partanen J, Tapaninaho A. Local denervation atrophy of paraspinal muscles in postoperative failed back syndrome. Spine 1993;18:575-581.

21. Haig AJ, Tong HC, Kendall R. The bent spine syndrome: myopathy + biomechanics $=$ symptoms. Spine J 2006;6:190-194. 